\title{
Welcome all to the year 2012
}

\author{
Nadia Magnenat-Thalmann
}

Published online: 25 November 2011

(C) Springer-Verlag 2011

First of all, I would like to thank all authors for their strong interest and participation in The Visual Computer journal. Between the 1st of November 2010 and the 31st of October 2011, we received 149 original research submissions. We accepted 30 papers out of these 149 submissions. The acceptance rate is $20.13 \%$. I take this opportunity to ask authors to cite The Visual Computer papers when writing their own papers. Our journal meets high standards but needs to be more cited. It is then important to cite each time excellent published papers in The Visual Computer.

This year, we have some changes in the editorial board members. I take this opportunity to thank the following members for their constant commitment and hard work for the journal:

- Prof. Arie Kaufman

- Prof. David F. Rogers

- Prof. John A. Vince

- Prof. Julie Dorsey

- Prof. Helmut Pottmann

- Prof. Martin Isenburg

- Prof. Ron Fedkiw

- Prof. Sung Yong Shin

- Prof. Tsusaka Noma

- Prof. Yoshihisa Shinagawa

New members have joined the editorial board upon our invitations and I would like to welcome them:

- Prof. Ahmad H. Nasri (American University of Beirut, Lebanon)

- Prof. Eftychios Sifakis (University of Wisconsin-Madison, USA)

N. Magnenat-Thalmann ( $\varangle)$

MIRALab, University of Geneva, Geneva, Switzerland

e-mail: visualcomputer@miralab.ch
- Prof. Ingemar J. Cox (University College London, UK)

- Prof. Seungyong Lee (Pohang University of Science and Technology, South Korea)

- Prof. Sung-Eui Yoon (Korea Advanced Institute of Science and Technology, South Korea)

For the coming year, we schedule the following issues:

- January: Regular Issue

- February: Regular Issue

- March: Regular Issue

- April: Regular Issue

- May: Regular Issue

- June-August: Special Issue: CGI 2012 (Triple Special Issue, Computer Graphics International 2012, Bournemouth University, UK)

- September: Special Issue: 3DOR 2011 (Eurographics Workshop on 3D Object Retrieval 2011, Llandudno, UK)

- October: Special Issue: SIBGRAPI 2011 (Conference on Graphics, Patterns and Images 2011, Alagoas, Brazil)

- November: Special Issue: CAD/Graphics 2011 (International Conference on CAD/Graphics 2011, Jinan, China)

- December: Regular Issue

I would like warmly to thank our reviewers for the tremendous jobs they do to carefully review papers. Without devoted reviewers, no paper could be published. You will find below the list of reviewers who kindly accepted to review The Visual Computer papers.

I would like also to thank the publisher, Ms Beverley Ford for her constant support. I also appreciate the collaboration of Beate Uhl, Rachel Roberts and Ronila Gamatero at Springer on a daily basis. It is really a pleasure to work with Springer people who are so dedicated to the journal and help to solve many issues.

Finally, I would like to introduce our new associate editor-in-chief, Prof. Shi-Min Hu, from Tsinghua Univer- 
sity. He will help greatly to increase the ranking of the journal by collaborating on the selection of the reviewers, reducing the reviewing time and proposing special issues of utmost interest.

I wish all of you a Happy New Year, a lot of academic success and a good health.

\section{Nadia Magnenat-Thalmann} Editor-in-Chief

\section{Reviewers List}

Alexander Agathos, Institute of Informatics and Telecommunications, Greece

Ceyhun Akgul, Vistek-ISRA Vision, Turkey

Karteek Alahari, INRIA-Willow Project, France

Remi Allègre, LSIIT CNRS Research Lab, France

Brian Allen, Nanyang Technological University, Singapore

Gholamreza Anbarjafari, Cyprus International University, Turkey

Gennady Andrienko, Fraunhofer Institute for Intelligent Analysis and Information Systems, Germany

Boris Aronov, Polytechnic Institute of NYU, USA

Ulf Assarsson, Chalmers University of Technology, Sweden

Kalle Astrom, Lund University, Sweden

Dominique Attali, CNRS, France

Marco Attene, Institute for Applied Mathematics and Information Technologies, Italia

Oscar Kin-Chung Au, City University of Hong Kong, Hong Kong

Basile Audoly, CNRS, France

Hernan Badino, Carnegie Mellon University, USA

Xue Bai, Adobe Systems, USA

Werner Bailer, Institute of Information Systems and Information Management, Austria

Hujun Bao, Zhejiang University, China

Jernej Barbic, University of Southern California, USA

Gill Barequet, Technion-Israel Institute of Technology, Israel

Loic Barthe, Paul Sabatier University, France

Harlen Costa Batagelo, State University of Campinas, Brazil

Philippe Bekaert, Hasselt University, Belgium

Mirela Ben-Chen, Stanford University, USA

Boaz Ben-Moshe, Ben-Gurion University of the Negev, Israel

Carsten Benthin, Intel's Advanced Graphics Lab, USA

Stefano Berretti, University of Florence, Italy
Martin Bertram, TU Kaiserslautern, Germany

John Biddiscombe, Swiss Federal Institute of Technology Zurich, Switzerland

Andrea Bottino, Polytechnic University of Turin, Italy

Tamy Boubekeur, TELECOM ParisTech, France

Pierre Boulanger, University of Alberta, Canada

Kévin Boulanger, INRIA Rennes, France

Ken Brodlie, University of Leeds, UK

Michael Brown, National University of Singapore, Singapore

Benedict Brown, Katholieke Universiteit Leuven, Belgium

Stefan Bruckner, Vienna University of Technology, Austria

Martin Cadik, Max Planck Institute for Computer Science, Germany

Marco Callieri, IFC-CNR, Italia

Mario Fernando Montenegro Campos, Federal University of Minas Gerais, Brazil

Yong Cao, Virginia Tech, USA

Nathan A. Carr, Adobe Systems, USA

Ming-Ching Chang, GE Global Research, USA

Wei Chen, Zhejiang University, China

Xuejin Chen, Yale Computer Graphics, China

Xiaobai Chen, Princeton University, USA

Sunghyun Cho, Pohang University of Science and Technology, Republic of Korea

Yi-King Choi, University of Hong Kong, Hong Kong

Niels Jørgen Christensen, Technical University of Denmark, Denmark

Nelson Chu, Microsoft Research, USA

Esteban Clua, Fluminense Federal University, Brazil

David Cohen-Steiner, INRIA, France

Forrester Cole, Massachusetts Institute of Technology, USA

João Comba, Federal University of Rio de Janeiro, Brazil

Mohamed Daoudi, TELECOM Lille1, France

Sukhendu Das, Indian Institute of Technology Madras, India

Tomas Davidovic, Universitaet Des Saarlandes, Germany

Paul Debevec, University of Southern California, USA

Ricard Delgado-Gonzalo, École Polytechnique Fédérale de Lausanne, Switzerland

Zhigang Deng, University of Houston, USA

Fabian Di Fiore, Hasselt University, Belgium

Yuanyuan Ding, Epson Research and Development, Inc., USA

Meng Ding, Zhejiang University, China

Bekir Dizdaroglu, Karadeniz Technical University, Turkey 
Zhao Dong, Cornell University, USA

Ye Duan, University of Missouri, USA

Mustafa Tolga Eren, Sabancı University, Turkey

Thomas Ertl, University of Stuttgart, Germany

William Evans, University of British Columbia, Canada

Jialue Fan, Northwestern University, USA

Ricardo Cordeiro de Farias, Federal University of Rio Grande do Sul, Brazil

Xinhuan Feng, Sichuan University, China

Danyel Fisher, Microsoft Research, USA

Shachar Fleishman, University of Utah, USA

Karl-Ingo Friese, University of Hanover, Germany

Hongbo Fu, City University of Hong Kong, Hong Kong

Chi-Wing Fu, Hong Kong University of Science and Technology, Hong Kong

Orazio Gallo, University of California, Santa Cruz, USA

Fabio Ganovelli, ISTI-CNR, Italy

Andrea Giachetti, Verona University, Italy

Daniela Giorgi, Institute for Applied Mathematics and Information Technologies, Italy

Marc Glisse, INRIA, France

Joao Paulo Gois, Federal University of ABC, Brazil

Boqing Gong, Chinese University of Hong Kong, Hong Kong

Xianfeng David Gu, State University of New York at Stony Brook, USA

Laura Gui, University of Geneva, Switzerland

Yanwen Guo, Nanjing University

Ralf Habel, Vienna University of Technology, Austria

Stefanie Hahmann, INRIA Rhone Alpes, France

Ying He, Nanyang Technological University, Singapore

Kaiming He, Microsoft Research Asia, China

Rachel Heck, University of Wisconsin, USA

Elsayed Hemayed, Cairo University, Egypt

Klaus Hildebrandt, Free University of Berlin, Germany

Matthias Hollander, Telecom ParisTech, France

Jeong-Mo Hong, Korea University, Republic of Korea

Kai Hormann, University of Lugano, Switzerland

Alexander Hornung, Disney Research Zurich, Switzerland

Ingrid Hotz, Zuse Institute Berlin, Germany

Donald H. House, Clemson University, USA

John Howat, Carleton University, Canada

Yiqun Hu, University of Western Australia, Australia

Qixing Huang, Stanford University, USA
Jiwu Huang, Sun Yat-sen University, China

Haidi Ibrahim, University of Science Malaysia, Malaysia

Yoshihisa Ijiri, Nagoya University, Japan

Thiago Ize, University of Utah, USA

Pranam Janney, University of New South Wales, Australia

Adrian Jarabo, University of Zaragoza, Spain

Krivanek Jaroslav, Charles University, Prague, Czech Republic

Dong Hyun Jeong, University of the District of Columbia, USA

Hui Ji, National University of Singapore, Singapore

Hongjun Jia, University of North Carolina, USA

Zhen Jia, United Technologies Research Center, China

Jiaya Jia, Chinese University of Hong Kong, Hong Kong

Yuntao Jia, Facebook, USA

Xiaogang Jin, Zhejiang University, China

Tao Ju, Washington University in St. Louis, USA

Tilke Judd, Massachusetts Institute of Technology, USA

Ioannis Kakadiaris, University of Houston, USA

Marcelo Kallmann, University of California, USA

Hongwen Kang, Carnegie Mellon University, USA

Craig Kaplan, University of Waterloo, Canada

Mustafa Kasap, University of Geneva, Switzerland

Ali Kemal Sinop, Carnegie Mellon University, USA

Ryan Kennedy, University of Pennsylvania, USA

John Keyse, Texas A\&M University, USA

Gu-jin Kim, Kyungpook National University, Republic of Korea

Doyub Kim, Seoul National University, Republic of Korea

Tae-hoon Kim, Korea Advanced Institute of Science and Technology, Republic of Korea

Myung-Soo Kim, Seoul National University, Republic of Korea

Chang-Hun Kim, Korea University, Republic of Korea

ByungMoon Kim, NVIDIA, USA

Hansung Kim, University of Surrey, UK

Gerald Jounghyun Kim, Korea University, Republic of Korea

Gordon Kindlmann, University of Chicago, USA

Robert Kosara, UNC Charlotte, USA

Jens Krueger, Technical University Munich, Germany

Yau-Hwang Kuo, National Cheng Kung University, Taiwan, Republic of China

Yu-Kun Lai, Cardiff University, UK 
Yu-Chi Lai, National Taiwan University of Science and Technology, Taiwan, Republic of China

Eric Landreneau, Texas A\&M University, USA

Longin Jan Latecki, Temple University, USA

Ken Law, City University of Hong Kong, Hong Kong

Kang Hoon Lee, Kwangwoon University, Republic of Korea

In-Kwon Lee, Yonsei University, Republic of Korea

Yunjin Lee, Ajou University, Republic of Korea

Geehyuk Lee, Korea Advanced Institute of Science and Technology (KAIST), Republic of Korea

Howard Leung, City University of Hong Kong, Hong Kong

Chi Sing Leung, City University of Hong Kong, Hong Kong

Thomas Lewiner, Pontifical Catholic University of Rio de Janeiro, Brazil

J.P. Lewis, Interval Research Corporation, USA

Ming Li, Unilever R\&D, UK

Bo Li, Nanyang Technological University, Singapore

Kuiyu Li, Ohio State University, USA

Guangda Li, National University of Singapore, Singapore

Xian-Ying Li, Tsinghua University, China

Wen-Chieh (Steve) Lin, Carnegie Mellon University, USA

Zhouchen Lin, Microsoft Research Asia, China

Weiyao Lin, Shanghai Jiao Tong University, China

Xiaopei Liu, Nanyang Technological University, Singapore

Baoquan Liu, University Bedfordshire, UK

Ligang Liu, Zhejiang University, China

Xinguo Liu, Zhejiang University, China

Shiguang Liu, Tianjin University, China

Zicheng Liu, Microsoft Research, USA

Yang Liu, Microsoft Research Asia, China

Alfredo Liverani, University of Bologna, Italy

Céline Loscos, University of Girona, Spain

Guojun Lu, Monash University, Australia

Xiaohan Ma, University of Houston, USA

Ross Maciejewski, Arizona State University, USA

Anderson Maciel, Federal University of Rio de Janeiro, Brazil

Marcus Magnor, TU Braunschweig, Germany

Khaled Mamou, AMD, France

Stephan Mantler, VRVis Research Center, Austria

Simone Marini, Institute for Applied Mathematics and Information Technologies, Italy

Ricardo Guerra Marroquim, Federal University of Rio de Janeiro, Brazil
Ralph Martin, Cardiff University, UK

Dimas Martinez, Federal University of Alagoas, Brazil

André Maximo, National Institute for Pure and Applied Mathematics, Brazil

Marie-Laurence Mazure, Joseph Fourier University, France

Pooran Memari, California Institute of Technology, USA

Daniel Meneveaux, University of Poitiers, France

David Menotti, Federal University of Ouro Preto, Brazil

Paul Merrell, Stanford University, USA

Rosane Minghim, University of São Paulo, Brazil

Ajay Mishra, University of Maryland, USA

Joseph S.B. Mitchell, State University of New York at Stony Brook, USA

Brendan Moloney, Oregon Health \& Science University, USA

Uma Mudengudi, B.V. Bhoomaraddi College of Engineering and Technology, USA

Heinrich Mueller, Dortmund University of Technology, Germany

Soraia Musse, Pontifical Catholic University of Rio Grande do Sul, Brazil

P.J. Narayanan, International Institute of Information Technology, India

Gabriele Nataneli, University of California, Los Angeles, USA

Laszlo Neumann, University of Girona, Spain

Junyong Noh, Korea Advanced Institute of Science and Technology (KAIST), Republic of Korea

Marc Olano, University of Maryland, USA

Antonio Alberto Oliveira, Federal University of Rio de Janeiro, Brazil

Manuel M. Oliveira, Federal University of Rio Grande do Sul, Brazil

Carol O'Sullivan, Trinity College Dublin, Ireland

Steve Yann Oudot, INRIA, France

Frank Pagel, Fraunhofer Institute for Optronics, System Technologies and Image Exploitation, Germany

Pietro Pala, University of Florence, Italy

Igor Pandzic, University of Zagreb, Croatia

Daniele Panozzo, University of Genoa, Italy

George Papagiannakis, University of Crete, Greece

Georgios Papaioannou, Athens University of Economics and Business, Greece

Jinho Park, University of California, Los Angeles, USA

Sang Il Park, Sejong University, Republic of Korea

Alexander Pasko, Hosei University, Japan 
Vorapoj Patanavijit, Assumption University, Thailand

Giuseppe Patane', Institute for Applied Mathematics and Information Technologies, Italy

Kedar Patwardhan, GE Global Research, USA

Frédéric Payan, I3S (UNSA-CNRS), France

Shahram Payandeh, Simon Fraser University, Canada

Li Peihua, Heilongjiang University, China

Stavros Perantonis, Institute of Informatics and Telecommunications, Greece

Jeff M Phillips, University of Utah, USA

Robert Pless, Washington University, USA

Konrad Polthier, Free University of Berlin, Germany

Federico Ponchio, ISTI-CNR, Italy

Ioannis Pratikakis, Institute of Informatics and Telecommunications, Greece

Hartmut Prautzsch, University Medical Center HamburgEppendorf, Germany

Albert Pritzkau, University of Leipzig, Germany

Enrico Puppo, University of Genoa, Italy

Dongxu Qi, Macau University of Science and Technology, China

Xueying Qin, Zhejiang University, China

Huamin Qu, Hong Kong University of Science and Technology, Hong Kong

Bruno Raffin, INRIA, France

A.N. Rajagopalan, Indian Institute of Technology Madras, India

Shanmuganathan Raman, Indian Institute of Technology, India

Gabor Renner, Computer and Automation Research Institute, Hungarian Academy of Sciences, Hungary

Christof Rhemann, Vienna University of Technology, Austria

Tobias Ritschel, Max Planck Institute for Computer Science, Germany

Christian Roessl, Max Planck Institute for Computer Science, Germany

Przemyslaw Rokita, Warsaw University of Technology, Poland

Michael Rubinstein, Massachusetts Institute of Technology, USA

Filip Sadlo, University of Stuttgart, Germany

Anthony Santella, Rutgers University, USA

Radim Sara, Czech Technical University, Czech Republic

Hagit Schechter, University of British Columbia, Canada

Daniel Scherzer, Vienna University of Technology, Austria
Jens Schneider, King Abdullah University of Science and Technology, Saudi Arabia

Tobias Schreck, University of Konstanz, Germany

Thomas Schultz, Max Planck Campus in Tübingen, Germany

Michael Schwärzler, Vienna University of Technology, Austria

Pradeep Sen, University of New Mexico, USA

Jun Kyung Seong, University of Utah, USA

Ariel Shamir, Efi Arazi School of Computer Science, Israel

Shiguang Shan, Institute of Computing Technology, Chinese Academy of Sciences, China

Rajiv Sharma, Seoul National University, Republic of Korea

Roy Shi, Microsoft Research, USA

Hyun Joon Shin, Ajou University, Republic of Korea

Takaaki Shiratori, Disney Research Zurich, Switzerland

Dominik Sibbing, RWTH Aachen University, Germany

Eftychios D. Sifakis, University of Wisconsin-Madison, USA

Kok Swee Sim, Multimedia University, Malaysia

Kwang Won Sok, Seoul National University, Republic of Korea

Cyril Soler, INRIA, France

Gael Sourimant, IRISA, France

Richard Souvenir, UNC Charlotte, USA

Jonas Spillmann, Swiss Federal Institute of Technology Zurich, Switzerland

Marc Stamminger, University of Erlangen-Nuremberg, Germany

Olivier St-Cyr, York University, Canada

Carsten Stoll, Max Planck Institute for Computer Science, Germany

Selcuk Sumengen, Sabanci University, Turkey

Xianfang Sun, Cardiff University, UK

Chiew-Lan Tai, Hong Kong University of Science and Technology, Hong Kong

Yu-Wing Tai, Korea Advanced Institute of Science and Technology, Republic of Korea

Kenshi Takayama, University of Tokyo, Japan

Ping Tan, National University of Singapore, Singapore

Zheng-Hua Tan, Aalborg University, Denmark

Tiow Seng Tan, National University of Singapore, Singapore

Cheng-Yuan Tang, Huafan University, Taiwan, Republic of China 
Kai Tang, Hong Kong University of Science and Technology, Hong Kong

Min Tang, Zhejiang University, China

Marco Tarini, ISTI-CNR, Italy

Franco Tecchia, University College London, UK

Frank Ter Haar, TNO-innovation for life, Netherlands

Theoharis Theoharis, University of Athens, Greece

Philippe Thevenaz, École Polytechnique Fédérale de Lausanne, Switzerland

Jing Tian, Institute for Infocomm Research, Singapore

Robert F. Tobler, VRVis Research Center, Austria

Yiying Tong, Michigan State University, USA

Panos Trahanias, Institute of Computer Science, Foundation for Research and Technology Hellas, Greece

Nicholas Q. Tran, Santa Clara University, USA

Changhe Tu, Shandong University, China

Borom Tunwattanapong, University of Southern California, USA

Georg Umlauf, University of Applied Science Constance, Germany

Sebastien Valette, CREATIS-LRMN, France

Jean-Philippe Vandeborre, TELECOM Lille 1, France

Daniel Vaquero, University of California, Santa Barbara, USA

Remco Veltkamp, Utrecht University, Netherlands

Romain Vergne, University of Giessen, France

Thomas Lucas Viard, gocad research group_-ASGA, France

Paul M.B. Vitanyi, University of Amsterdam, Netherlands

Markus Wacker, Dresden University of Applied Sciences, Germany

Marcelo Walter, Federal University of Pernambuco, Brazil

Liang Wan, Tianjin University, China

Rui Wang, University of Massachusetts, USA

Yu-Ping Wang, Tsinghua University, Chna

Guoping Wang, Peking University, China

Oliver Wang, Disney Research Zurich, Switzerland

Lvdi Wang, Microsoft Research Asia, China

Zhen Wen, IBM T.J. Watson Research Center, USA

Michael Wimmer, Vienna University of Technology, Austria

Thomas Wischgoll, Wright State University, USA

Christian Wolf, University of Lyon, France
Zongmin Wu, Fudan University, China

Tai-Pang Wu, Hong Kong University of Science and Technology, Hong Kong

Stefanie Wuhrer, Saarland University, Germany

Zhifeng Xie, Shanghai Jiao Tong University, China

Zhiwei Xiong, University of Science and Technology of China, China

Weiwei Xu, Microsoft Research Asia, China

Wang Yanbin, Nanyang Technological University, Singapore

Xiaosong Yang, Bournemouth University, UK

Jing Yang, UNC Charlotte, USA

Ming-Hsuan Yang, University of California at Merced, USA

Yong-Liang Yang, King Abdullah University of Science and Technology, Saudi Arabia

Ji Soo Yi, Purdue University, USA

Shihui Ying, Shanghai University, China

Shin Yoshizawa, RIKEN, Japan

Qizhi Yu, University College London, UK

Xiaodong Yu, University of Maryland, USA

Xiaotong Yuan, National University of Singapore, Singapore

Cem Yuksel, Texas A\&M University, USA

Gabriel Zachmann, Clausthal University, Germany

Dirk Zeckzer, University of Kaiserslautern, Germany

Wei Zeng, Stony Brook University, USA

Guo-Xin Zhang, Tsinghua University, China

Richard (Hao) Zhang, Simon Fraser University, Canada

Hongxin Zhang, Zhejiang University, China

Eugene Zhang, Oregon State University, USA

Yanci Zhang, Sichuan University, China

Liuxin Zhang, Beijing Institute of Technology, China

Guofeng Zhang, Zhejiang University, China

Qiaoping Zhang, Intermap Technologies, China

Liming Zhao, D.E. Shaw Research, USA

Yao Zhao, Beijing Jiaotong University, China

Yicong Zhou, University of Macau, China

Zhiyu Zhou, Zhejiang Sci-Tech University, China

Yingxuan Zhu, GE Global Research, USA

Arno Zinke, University of Bonn, Germany 